\title{
Large Dumbbell-Shaped C1 Schwannoma Presenting as a Foramen Magnum Mass
}

\author{
Jody Helms, M.D. ${ }^{1}$ Lattimore Madison Michael II, M.D., F.A.A.N.S., F.A.C.S. ${ }^{1,2}$ \\ ${ }^{1}$ Department of Neurosurgery, University of Tennessee Health \\ Science Center, Memphis, Tennessee \\ 2 Semmes-Murphey Neurologic and Spine Institute, Memphis, \\ Tennessee

\begin{abstract}
Address for correspondence and reprint requests Lattimore Madison Michael II, M.D., F.A.A.N.S., F.A.C.S., Semmes-Murphey Clinic, 6325 Humphreys Blvd., Memphis, TN 38120

(e-mail: mmichael@semmes-murphey.com).
\end{abstract}

J Neurol Surg Rep 2012;73:32-36.
Abstract Keywords
- schwannoma
- C1
- first cervical nerve root
- dumbbell shape
- foramen magnum mass

\begin{abstract}
Schwannomas involving the foramen magnum commonly originate from the lower cranial nerves, but they are rarely found arising from the first cervical root. To date, very few cases have been described in the literature. The majority involve either the intradural or extradural compartment but not both. We report the second case of a dumbbell-shaped schwannoma arising from the first cervical root. Our patient presented with hemisensory deficits secondary to brainstem compression at the level of the foramen magnum. The patient underwent a far lateral approach, and a gross total resection was achieved. Preoperative suspicion of the diagnosis is helpful in anticipating displacement and avoiding damage to the surrounding neurovascular structures.
\end{abstract}

Schwannomas of the first cervical nerve root are extremely rare. Similar to other benign pathology occurring in the foramen magnum region, they commonly present with signs and symptoms of brainstem compression. Preoperative neuroradiological evaluation with computed tomography (CT) and magnetic resonance imaging (MRI) is incredibly useful in lending confidence to the diagnosis of a $\mathrm{C} 1$ schwannoma. Because of their origin along the course of the $\mathrm{C} 1$ nerve root, the adjacent neurovascular structures are displaced in a predictable fashion.

\section{Case Report}

A 29-year-old Caucasian woman was referred to the outpatient neurosurgical clinic for evaluation of a mass found at the craniocervical junction. Approximately 2 months before presentation, she began experiencing progressive numbness in her left arm and leg. She noticed that while she was breastfeeding her 6-month-old child, she had no sensation on the left side of her chest. She felt subjectively weak on the left side, but she remained independent and ambulatory. Her history was significant for non-Hodgkin's lymphoma, which was diagnosed at the age 11 . Following the diagnosis of lympho-

received

September 8, 2011

accepted after revision

November 23, 2011

published online

April 20, 2012 ma, she was treated with chemotherapy without radiation. The patient had been in remission since treatment as verified by annual laboratory testing.

Upon examination, she was awake and alert. Cranial nerve testing was completely normal. Strength was symmetric and full bilaterally. She had decreased sensation of the left hemibody to light touch and pinprick. Reflexes were normoactive. Cerebellar testing was nonfocal. A MRI scan of the brain with and without contrast revealed an extra-axial mass at the craniocervical junction asymmetric to the left. It measured $2.6 \times 2.3 \times 3.2 \mathrm{~cm}$ in greatest transverse, AP, and craniocaudal dimensions. The lesion exerted mass effect upon the brainstem displacing it to the right with compression. The mass was hyperintense on T2-weighted sequences, hypointense on T1-weighted sequences, and demonstrated avid enhancement (-Figs. 1-4). There was also extradural extension inferiorly below the vertebral artery but above the lamina of the first cervical lamina (-Fig. 5). No hydrocephalus was seen. A CT scan revealed marked thinning of the C1 lamina on the left side (-Fig. 6). A cerebral angiogram revealed the lesion to be largely hypovascular.

The operative plan included a left-sided far lateral approach for an attempted gross total removal of the lesion.
Copyright $\odot 2012$ by Thieme Medical Publishers, Inc., 333 Seventh Avenue, New York, NY 10001, USA. Tel: +1(212) 584-4662.
DOI http://dx.doi.org/ $10.1055 / \mathrm{s}-0032-1311757$ ISSN 2193-6358. 


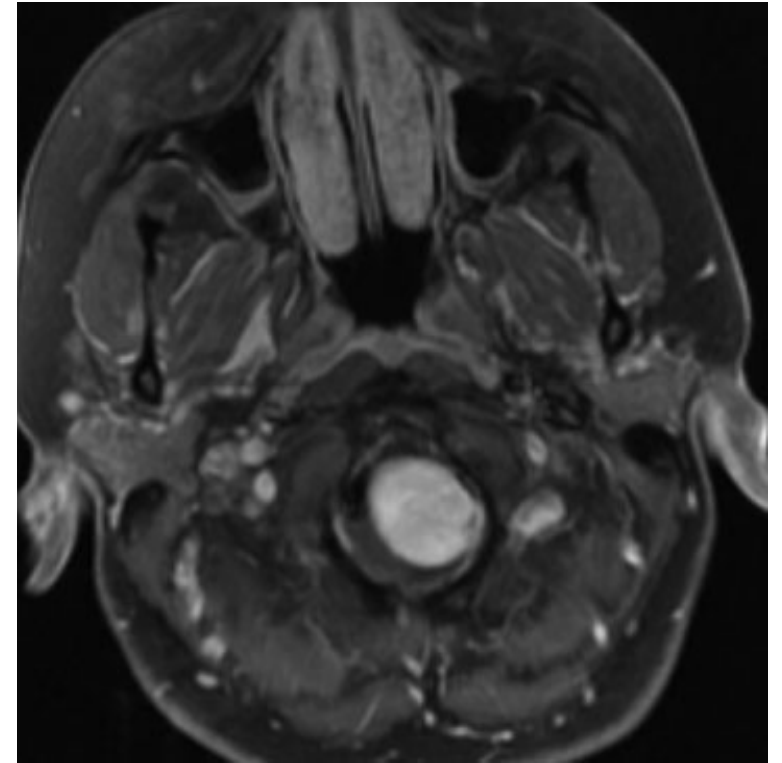

Figure 1 Preoperative axial T1 weighted magnetic resonance imaging with contrast demonstrates a homogenously enhancing mass within the foramen magnum.

Electrophysiological monitoring was not used as the tumor was expected to be extra-axial in location with a clear surgical plane. A lumbar drain was placed to facilitate relaxation of the neural elements during the operation. Her head was then placed in a cranial fixation system and it was aligned such as to maximally expose the left suboccipital region. A retroauricular C-shaped incision was performed. The incision began $\sim 2 \mathrm{~cm}$ superior to the pinna and extended posteriorly and

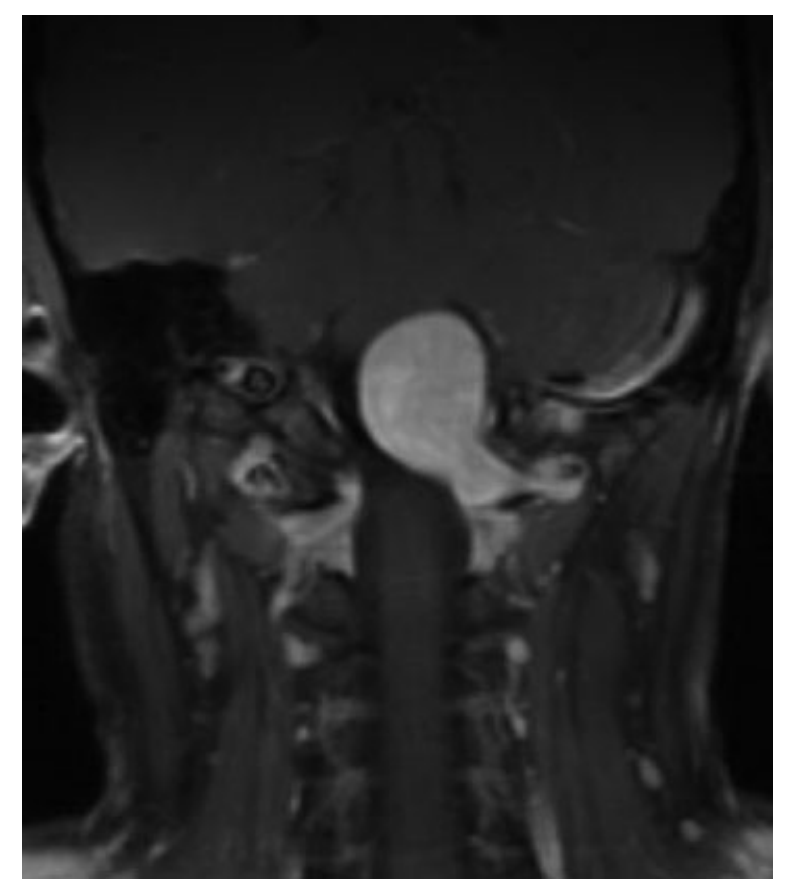

Figure 2 Preoperative coronal T1 weighted magnetic resonance imaging with contrast reveals dumbbell-shaped mass with extradural portion of the tumor coursing inferior to the vertebral artery.

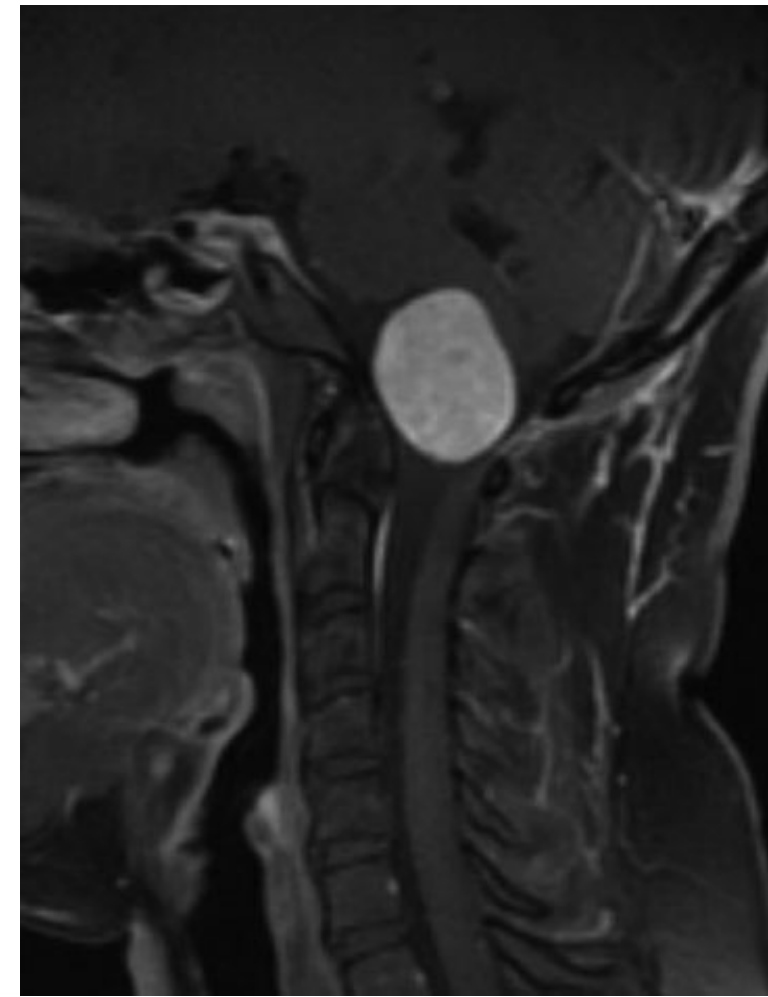

Figure 3 Preoperative sagittal T1 weighted magnetic resonance imaging with contrast showing marked displacement of the brainstem.

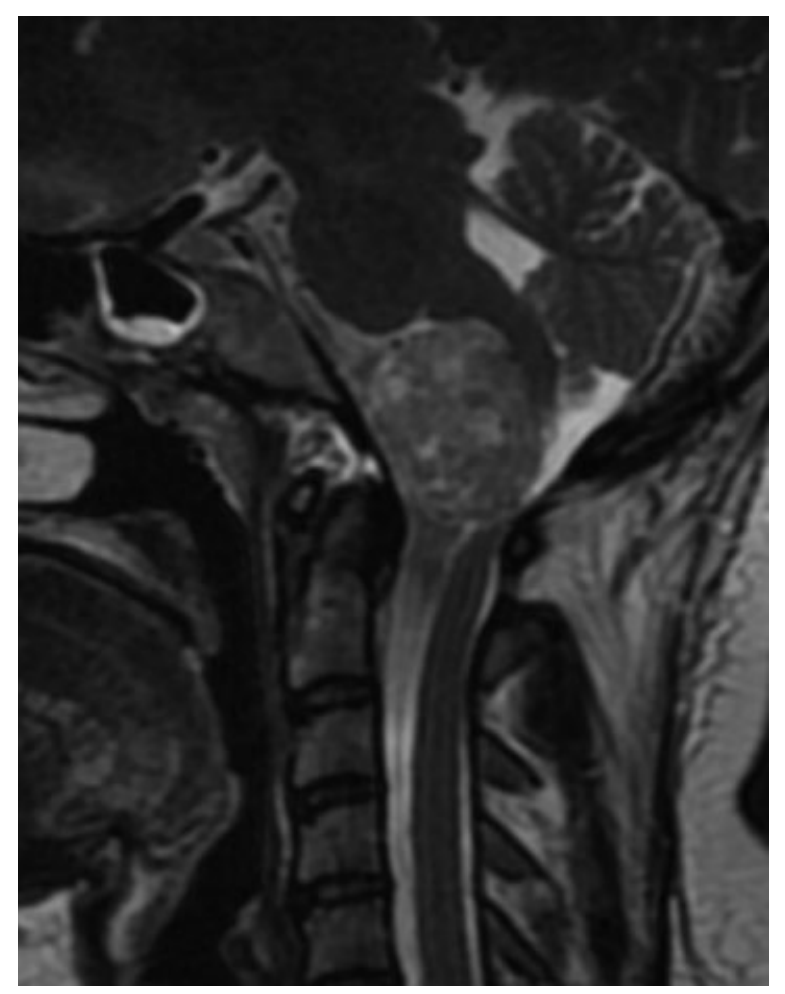

Figure 4 The lesion appears slightly hypointense to cerebrospinal fluid on the preoperative sagittal T2 weighted magnetic resonance imaging. 


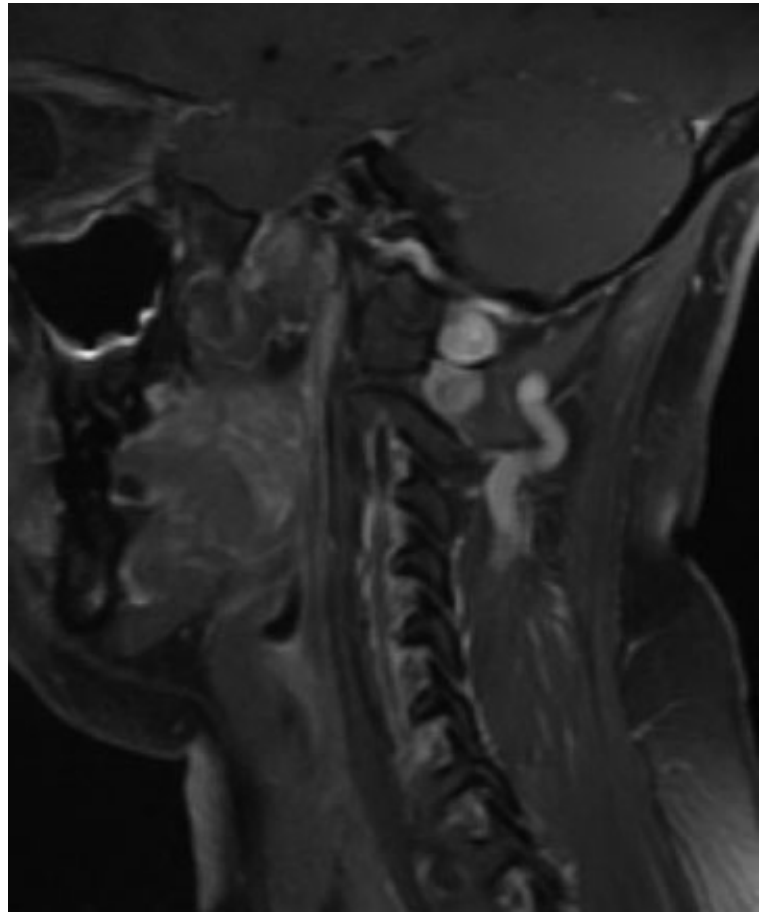

Figure 5 Preoperative sagittal T1 weighted magnetic resonance imaging with contrast revealing tumor along the lamina of $\mathrm{C} 1$.

then inferiorly to end in a pre-existing skin crease. The muscle flap superiorly was mobilized to expose the region of the asterion. The inferior muscle mass was mobilized inferiorly to expose the left skull base, left aspect of $\mathrm{C} 1$, and the left hemilamina of $\mathrm{C} 2$. The drill was then taken, and the inferior aspect of the left skull base was resected to the level of the occipital condyle. Owing to the fact that the tumor ascended superiorly, a transtemporal approach with partial mastoidectomy was performed to expose the sigmoid sinus and jugular

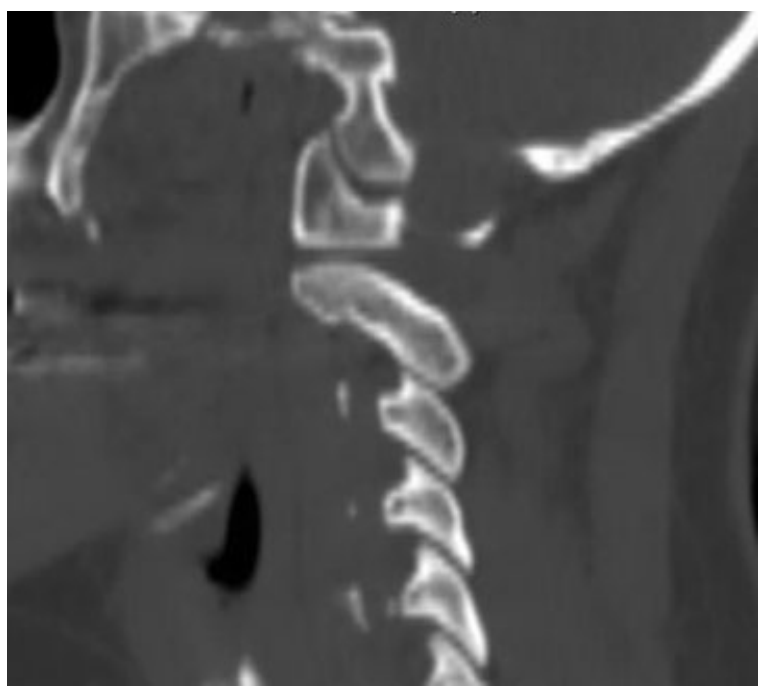

Figure 6 Erosion of the lamina of C1 is demonstrated on a preoperative sagittal computed tomography scan. bulb. The left hemilamina of $\mathrm{C} 1$ was resected in addition to a partial superior C2 laminectomy. The dura was opened in a linear fashion just posterior to the sigmoid sinus and jugular bulb region. It was carried inferiorly posterior to the dural penetration of the extradural vertebral artery across the craniocervical junction to the level of the superior aspect of C2. The tumor was immediately encountered.

On the surface of the tumor was the accessory nerve. An aggressive intratumoral debulking ensued following opening of the capsule. Once this was accomplished, one could visualize the structures around the tumor. The ninth and tenth nerves were seen superiorly. The eleventh nerve, as stated, was coursing along the posterior surface of the tumor mass. The brainstem region was seen on the medial aspect of the tumor, and it was markedly compressed. The vertebral artery was seen both anteriorly and superiorly. The tumor was then folded upon itself, and further removal was achieved. There was a cuff of tumor along the intradural vertebral artery, and this was sharply incised to the level of the adventitia. The only area in which there was attachment to the brainstem was at the level where $\mathrm{C} 1$ was coming off the neural trunk. This was incised sharply. The C2 nerve roots were investigated and were completely normal.

With the entire intradural tumor being removed, attention then turned to the extradural portion. The plane between the tumor and the extradural vertebral artery was developed. The vertebral artery was markedly compressed superiorly. Sharp dissection was used to develop this plane. A gross total removal of the tumor was verified at this time. The dura was closed primarily in the superior and inferior half of the dural opening. The middle portion, which consisted of a large dural defect at the level of the neural foramen, required the use of a free pericranial patch graft. The remainder of the wound was closed in layers.

Postoperatively, her neurological examination normalized with complete return of sensation on the left side of her body over several days. Pathology was consistent with a schwannoma, and imaging verified a gross total resection was achieved (-Fig. 7). She is now over 3 years from surgery, and her imaging reveals no residual or recurrent tumor.

\section{Discussion}

Schwannomas of the skull base region most commonly arise from the lower cranial nerves or the $\mathrm{C} 2$ nerve root. Schwannomas arising from $\mathrm{C} 1$ are very rare. In part, this can be attributed to the fact that schwannomas most commonly arise from dorsal sensory roots. $\mathrm{C} 1$ controls the function of the rectus capitis lateralis and, historically, was not thought to have a sensory component. ${ }^{1}$ In a recent anatomic study, Tubbs et $\mathrm{al}^{2}$ verified the presence of a sensory contribution in a portion of specimens. They identified C1 dorsal roots in $46.6 \%$ of specimens and $28.5 \%$ had an associated dorsal root ganglion. They also identified in $50 \%$ of patients a communication of the spinal accessory nerve with the dorsal rootlets of $\mathrm{C}$, supporting an initial observation by Ouaknine and Nathan. ${ }^{3}$ In cases where a communication was present, no sensory elements to $\mathrm{C} 1$ were found. It is likely that these anatomic 

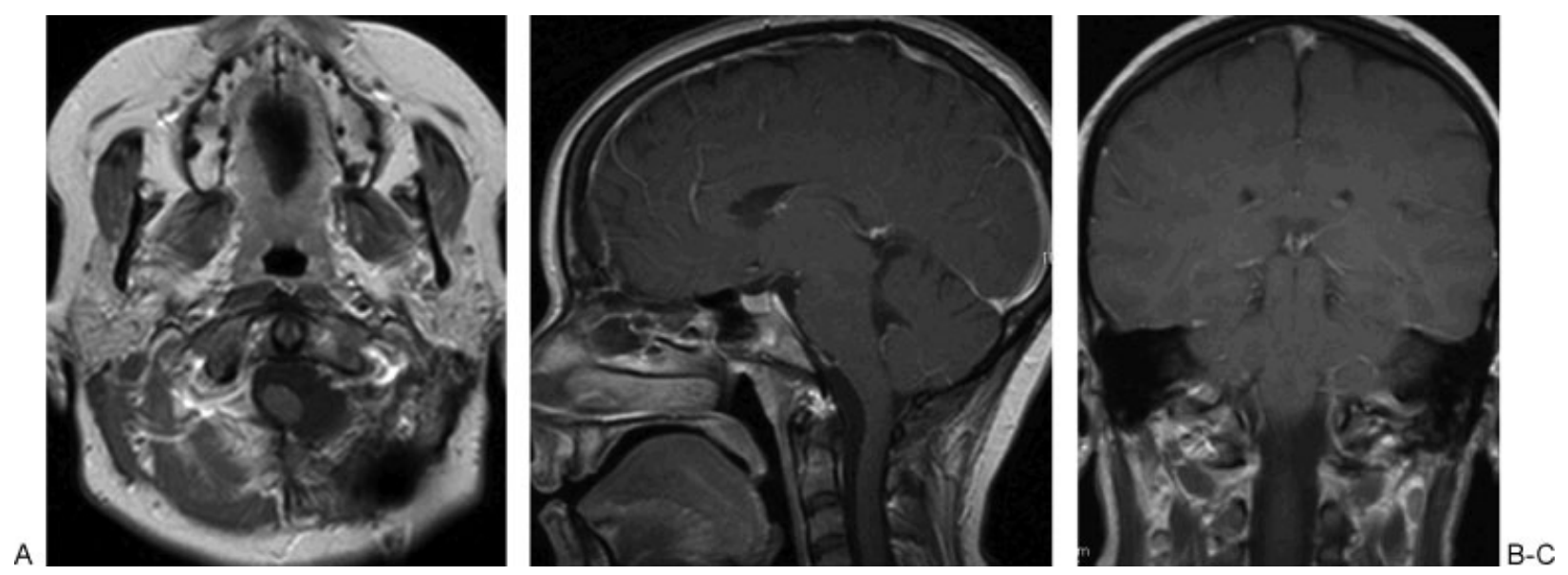

Figure 7 Postoperative (A) axial, (B) sagittal, and (C) coronal T1 weighted magnetic resonance imaging without contrast shows no evidence of residual neoplasm.

variations explain the rarity of $\mathrm{C} 1$ schwannomas relative to other locations.

To date, there have been few reports of C1-root schwannomas and only one case of a dumbbell-shaped one. In 1988, Guidetti and Spallone ${ }^{4}$ reported three cases of C-1 tumors, one of which was "hourglass" shaped and is the first and only clearly distinguished intradural/extradural C1 schwannoma reported. George and Lot $^{5}$ reported five cases of $\mathrm{C} 1$ neurinomas in 1995, but it is unclear how many of these lesions involved both the intradural and extradural compartments. Kalavakonda et $\mathrm{al}^{6}$ published in 2000 a single case report of an extradural C1 schwannoma causing symptomatic intermittent vertebral artery compression. In 2003, Parlato et al ${ }^{7}$ reported their management of benign craniovertebral junction tumors: out of 15 cases, 3 were $\mathrm{C} 1$ schwannomas but the location of the tumors was not described in the paper. One patient presenting with aggressive lateral mass invasion by an extradural C1 schwannoma was described by Kim et al in $2005 .{ }^{8}$ Three cases of intradural C1 schwannomas presenting as large foramen magnum lesions were reported by Ueda et al in 2006. ${ }^{9}$

It is beneficial to distinguish $\mathrm{C} 1$ schwannomas from meningiomas when assessing foramen magnum lesions (-Table 1). This can help with preoperative planning and predicting displacement of important neurovascular struc- tures. Symptom onset usually does not help, because both are slow growing and are ultimately discovered due to slow, increasing brainstem or upper cord compression. Imaging is invaluable. Computed tomography scans with bone windows often reveal thinning of the lamina of $\mathrm{C} 1$ secondary to widening of the $\mathrm{C} 1$ foramen in cases of either dumbbellshaped or extradural C1 schwannomas. Magnetic resonance imaging shows schwannomas with a more heterogeneous enhancement pattern whereas meningiomas are more homogenous with a broad-based dural attachment. ${ }^{10}$ With both meningiomas and schwannomas, the accessory nerve is displaced posteriorly and will be on the surface of the tumor following opening of the dura. As well, the lower cranial nerves will be displaced superiorly in both pathologies. With meningiomas, however, special care must be taken to ensure that there is a surgical plane between the tumor and the lower cranial nerves as it is well-established that tumor invasion is possible. In the case of schwannomas, a surgical plane will likely be present. As opposed to meningiomas, the intradural vertebral artery is consistently displaced laterally in the case of $\mathrm{C} 1$ schwannomas. The schwannoma is commonly located between the artery and the brainstem. In the extradural location, the vertebral artery will be displaced superiorly against the cranial base. Depending on the site of origin for

Table 1 Comparison of Foramen Magnum Meningiomas and C1 Schwannomas

\begin{tabular}{|l|l|l|}
\hline & Foramen Magnum Meningioma & C1 Schwannoma \\
\hline Symptom onset & Chronic & Chronic \\
\hline Relevant computed tomography findings & None & Thinning of C1 lamina \\
\hline Magnetic resonance imaging contrast pattern & Homogenous enhancement & Heterogenous enhancement \\
\hline Dural tail & Yes & No \\
\hline Accessory nerve location & Posterior & Posterior \\
\hline Lower Cranial nerve location & Superior & Superior \\
\hline C2 nerve root location & Varied location depending on origin & Posterior \\
\hline Intradural vertebral artery location & Varied location depending on origin & Lateral \\
\hline Cranial nerve invasion & Possible & Never \\
\hline
\end{tabular}


a meningioma, the location of the $\mathrm{C} 2$ nerve root is variable while they are expected to be displaced posteriorly in C1 schwannomas.

When anterior visualization of the upper cervical cord and medulla is needed, a far lateral approach gives the most direct route without the need for neural retraction. Midline posterior approaches to the anterior portion of the foramen magnum carry the disadvantage of interposing the neurovascular structures between the ventral tumor and the surgeon, possibly necessitating the use of unwanted retraction. As in this case, we feel that resection of the condyle with the far lateral approach is rarely needed as benign pathology often forms a wide posterolateral window to accomplish total tumor removal. If condyle removal is required, limiting the resection to the posterior third is desired to avoid hypermobility of the craniocervical junction. ${ }^{11}$

As was done in the present case, a gross total resection of C1 schwannomas is the goal. Individual anatomy must be considered to ensure this can be achieved safely.

\section{Conclusion}

We report the second case of a dumbbell-shaped schwannoma arising from the first cervical root. A far lateral approach was utilized to obtain a gross total resection. C1 schwannomas are rare tumors that should be considered in the differential of foramen magnum lesions. Preoperative suspicion of the diagnosis is helpful in anticipating displacement and avoiding damage to the surrounding neurovascular structures.

\section{References}

1 Rhoton ALJr. Cranial Anatomy and Surgical Approaches. Baltimore, MD: Lippincott Williams \& Wilkins; 2003:600

2 Tubbs RS, Loukas M, Yalçin B, Shoja MM, Cohen-Gadol AA. Classification and clinical anatomy of the first spinal nerve: surgical implications. J Neurosurg Spine 2009;10(4):390-394

3 Ouaknine G, Nathan H. Anastomotic connections between the eleventh nerve and the posterior root of the first cervical nerve in humans. J Neurosurg 1973;38(2):189-197

4 Guidetti B, Spallone A. Benign extramedullary tumors of the foramen magnum. Adv Tech Stand Neurosurg 1988;16:83-120

5 George B, Lot G. Neurinomas of the first two cervical nerve roots: a series of 42 cases. J Neurosurg 1995;82(6):917-923

6 Kalavakonda C, Sekhar LN, Jones RV, Rehaman AB. Intermittent vertebral artery compression caused by $\mathrm{C} 1$-root schwannoma: case report. Neurol Res 2000;22(7):679-684

7 Parlato C, Tessitore E, Schonauer C, Moraci A. Management of benign craniovertebral junction tumors. Acta Neurochir (Wien) 2003;145(1):31-36

$8 \mathrm{Kim}$ JH, Lee JH, Park YK, Kwon TH, Chung HS. C-1 root schwannoma with aggressive lateral mass invasion. Yonsei Med J 2005;46(4): 575-578

9 Ueda R, Yoshida K, Kawase T. Intradural C-1 ventral root schwannomas treated by surgical resection via the lateral suboccipital transcondylar approach-three case reports. Neurol Med Chir (Tokyo) 2006;46(6):298-301

10 Osborn AG. Tumors, cysts, and tumorlike lesions of the spine and spinal cord. In: Osborn AG. Diagnostic Neuroradiology. St Louis, MO: Mosby; 1994:895-900

11 Vishteh AG, Crawford NR, Melton MS, Spetzler RF, Sonntag VK, Dickman CA. Stability of the craniovertebral junction after unilateral occipital condyle resection: a biomechanical study. J Neurosurg 1999;90(1, Suppl):91-98 